\title{
Hadad-Bassagasteguy flap in skull base reconstruction - current reconstructive techniques and evaluation of criteria used for qualification for harvesting the flap
}

\author{
Piotr Wardas ${ }^{1,2}$, Michał Tymowski $^{3}$, Agnieszka Piotrowska-Seweryn ${ }^{1}$, Jarosław Markowski ${ }^{1}$, Piotr Ładziński ${ }^{3}$ \\ ${ }^{1}$ Clinical Department of Laryngology, School of Medicine in Katowice, Medical University of Silesia, Katowice, Poland \\ ${ }^{2}$ Department of Laryngology and ENT Oncology, Regional Hospital No. 5, Sosnowiec, Poland \\ ${ }^{3}$ Department of Neurosurgery, School of Medicine in Katowice, Medical University of Silesia, Regional Hospital No. 5, Sosnowiec, Poland
}

\begin{abstract}
Introduction: Cerebrospinal fluid (CSF) leakage is a common consequence or complication in the operations of skull base tumors. The Hadad-Bassagasteguy flap (HBF) is the most common local flap used in the reconstruction of the meninges. It is a nasoseptal flap (NSF) vascularized by the sphenopalatine artery (SPA). Improvement of the already existing techniques is necessary.

Aim: To present our experience in HBF and to evaluate the criteria used for qualification (relative and absolute indications) for the NSF reconstructive technique.

Material and methods: The retrospective study included 25 patients who underwent expanded endonasal approach (EEA) operations with the NSF. The correctness of qualification based on our own criteria was assessed. The most important modifications of the original HBF as well as the reasons for failures are discussed.

Results: There were 12 relative and 13 absolute indications for NSF harvesting. In 2 cases no anticipated CSF leakage was observed. No complications were reported.

Conclusions: Skull base reconstruction with HBF and its various modifications is a highly effective technique. Absolute indications for NSF harvesting prior to resection are: reoperations in the case of a previous open approach, preoperative CSF leakage, intradural localization of a tumor related to its etiopathogenesis, suspicion of intradural diffusion of a neoplasm in magnetic resonance imaging if the etiopathogenesis cannot clarify the tumor's relation to the meninges. Relative indications concern mostly pituitary macroadenomas of at least $2.5 \mathrm{~cm}$ in diameter.
\end{abstract}

Key words: cerebrospinal fluid, endoscopy, skull base, vascularized nasoseptal flap.

\section{Introduction}

The endonasal reconstructive techniques of skull base defects were initially performed in order to fix cerebrospinal fluid (CSF) leakage of mostly posttraumatic (including iatrogenic) etiology. Skull base reconstructions as an element of planned surgical treatment of lesions localized in this region have been developed as a consequence of new, wide approaches such as ex- panded endonasal approach (EEA) or endonasal skull base surgery (ESBS). Oscar Hirsch was the first author to report a successful closure of CSF leak by endonasal surgery, in 1952 [1]. However, it was Malte Wigand who performed pioneering endoscopic rhinorrhea closure with the application of a free mucosal flap in 1981 [2].

Until now, many different reconstructive techniques with the application of various autologous

\section{Address for correspondence}

Piotr Wardas, Clinical Department of Laryngology, School of Medicine, Medical University of Silesia, 20/24 Francuska St, 40-027 Katowice, Poland, e-mail: piotrwardas@onet.pl 
and synthetic materials have been presented. Operations with free flaps or vascular pedicled flaps comprise a large group of surgical procedures in skull base reconstruction. Among vascularised flaps, those raised locally in the nasal cavity from the nasal septum or nasal turbinates on the supplying sphenopalatine artery (SPA) are the most common. Pedicled flaps raised on external head and neck vessels other than intranasal ones are applied in situations when the SPA is damaged due to a tumor or previous treatment (surgical procedure or radiotherapy) [3]. The following flaps can be used: tunneled temporoparietal fascia flap, endoscopic-assisted pericranial flap, palatal flap, occipital flap, facial artery buccinator flap and others [3]. However, among all above options the vascularized pedicled nasoseptal flap (NSF) seems to be the 'gold standard' flap in the reconstruction of the meninges of the cranial base $[4,5]$. It was first described in 2006 by Hadad et al. and Luis Bassagasteguy, and it is also known as the Hadad-Bassagasteguy flap (HBF) [4]. The mentioned authors presented the first series of 43 operations with only $5 \%$ being failures (CSF leaks) [4]. In 2008 Kassam et al. found NSF extremely useful and reliable, reporting on a group of 75 patients with a $10.66 \%$ failure rate in the first 25 operated patients and only a 4\% CSF leak rate in the next 50 [6].

\section{Aim}

The aim of the study was to present our experience in the reconstructive technique of cranial base defects with application of the HBF. The authors discuss different aspects of harvesting techniques, flap implantation and modifications of the original procedure, analyzing the potential factors influencing the outcome of the operations, including failures. They compare their initial results to other authors' reports.

\section{Material and methods}

Patients who underwent an EEA operation due to skull base tumors in the neurosurgical department in the period 2014-2017 were identified. Medical records were reviewed for patient demographic information, pathology, CSF leak, indications for flap harvesting, NSF application, lumbar drainage and reoperations. The basic socio-clinical characteristics of the group are presented in Table I.
All patients $(n=25)$ underwent an EEA operation performed by the same team of ENT surgeon and neurosurgeon. The assessment of the probability of an intraoperative CSF leak was made for each case individually. The authors defined their own relative and absolute indications for NSF harvesting. The absolute indications were: reoperations in the case of a previous open approach, preoperative CSF leakage, intradural localization of a tumor related to its etiopathogenesis, suspicion of intradural diffusion of a neoplasm in magnetic resonance imaging (MRI) if the etiopathogenesis cannot clarify tumors' relation to meninges (mainly chordoma). Relative indications concerned mostly pituitary macroadenomas and they were based on tumor size according to Schwartz classification and localization [7]. The NSF harvesting was indicated in the case of macroadenoma of at least $2.5 \mathrm{~cm}$ in diameter.

The final outcome of the HBF reconstruction was analyzed. The study data were compared with other authors' findings, including the differences in percentage share of the particular types of the operated skull base pathologies.

\section{Results}

A retrospective review yielded 23 patients who underwent EEA operations with the application of NSF. According to the adopted criteria (described above), there were 12 relative and 13 absolute indications for NSF harvesting. In two cases no anticipated CSF leakage was observed; therefore there was no necessity of reconstruction of the meninges. In these cases the harvested flap was replanted to the donor site on the nasal septum. Among 23 NSF reconstructions there were 23 successful closures of the meninges lesion. One failure, i.e. CSF leakage in the first few days postoperatively, was noted. The patient required secondary repair and lumbar drainage was used for 7 days. The final successful outcome was observed in all 23 patients with NSF reconstruction. No meningitis or other early complications were observed. The above data are presented in Table I. An example of step-by-step NSF harvesting is shown in Photos 1-6.

\section{Discussion}

The final success of EEA operations with nasoseptal flaps depends not only on the surgical technique, but on many different factors. The lo- 
Table I. Socio-clinical characteristics of the study group

\begin{tabular}{|c|c|c|c|c|c|c|c|c|}
\hline No. & Gender & Age & Diagnosis & $\begin{array}{l}\text { Date of } \\
\text { operation }\end{array}$ & $\begin{array}{l}\text { Indications } \\
\text { for NSF } \\
\text { harvesting }\end{array}$ & $\begin{array}{l}\text { Flap } \\
\text { applica- } \\
\text { tion }\end{array}$ & $\begin{array}{l}\text { Lumbar } \\
\text { drainage }\end{array}$ & $\begin{array}{l}\text { Reopera- } \\
\text { tion }\end{array}$ \\
\hline 1 & $\mathrm{~F}$ & 36 & Neurinoma of NC V (reoperation) & 23.10.2014 & D & Y & $N$ & $N$ \\
\hline 2 & M & 63 & $\begin{array}{l}\text { Pituitary macroadenoma (grade } \\
\text { IV in Knosp classification) }\end{array}$ & 16.12 .2014 & $\mathrm{D}$ & Y & $N$ & $N$ \\
\hline 3 & M & 54 & Chordoma & 16.01.2015 & $\mathrm{D}$ & Y & $\mathrm{N}$ & $\mathrm{N}$ \\
\hline 4 & F & 55 & Fibrous dysplasia & 22.05 .2015 & I & N & $N$ & $N$ \\
\hline 5 & $\mathrm{~F}$ & 48 & $\begin{array}{c}\text { Pituitary adenoma (grade } 0 \text { in } \\
\text { Knosp classification) }\end{array}$ & 29.05 .2015 & I & $N$ & $N$ & $N$ \\
\hline 6 & M & 59 & Chordoma & 12.06 .2015 & $D$ & Y & $\mathrm{N}$ & $\mathrm{N}$ \\
\hline 7 & M & 54 & Chordoma & 2.10 .2015 & $D$ & Y & Y & $\begin{array}{c}Y \\
(12.10 .2015)\end{array}$ \\
\hline 8 & $\mathrm{~F}$ & 50 & Chordoma & 16.10.2015 & I & Y & $\mathrm{N}$ & $\mathrm{N}$ \\
\hline 9 & F & 69 & $\begin{array}{c}\text { Pituitary macroadenoma (grade } 0 \\
\text { in Knosp classification) }\end{array}$ & 4.12 .2015 & I & Y & $N$ & $N$ \\
\hline 10 & F & 33 & Rathke cleft cyst & 11.12 .2015 & I & Y & $N$ & $N$ \\
\hline 11 & $M$ & 44 & $\begin{array}{c}\text { Pituitary macroadenoma (grade } 0 \\
\text { in Knosp classification) }\end{array}$ & 22.01.2016 & I & Y & $N$ & $N$ \\
\hline 12 & M & 60 & Chordoma & 26.02 .2016 & D & Y & Y & $\mathrm{N}$ \\
\hline 13 & M & 26 & Hemangiopericytoma & 11.03 .2016 & I & Y & $\mathrm{N}$ & $N$ \\
\hline 14 & M & 40 & Chordoma & 20.05 .2016 & D & Y & $N$ & $N$ \\
\hline 15 & M & 37 & $\begin{array}{c}\text { Pituitary macroadenoma (grade } 1 \\
\text { in Knosp classification) }\end{array}$ & 3.06 .2016 & I & Y & $N$ & $N$ \\
\hline 16 & M & 40 & Chordoma & 8.07 .2016 & I & Y & $N$ & $N$ \\
\hline 17 & M & 68 & Pituitary macroadenoma & 20.10 .2016 & I & Y & $\mathrm{N}$ & $\mathrm{N}$ \\
\hline 18 & M & 61 & $\begin{array}{l}\text { Pituitary macroadenoma (grade III } \\
\text { in Knosp classification) }\end{array}$ & 25.11.2016 & I & Y & $\mathrm{N}$ & $\mathrm{N}$ \\
\hline 19 & $\mathrm{~F}$ & 52 & Petroclival meningioma & 25.08 .2017 & D & Y & $\mathrm{N}$ & $\mathrm{N}$ \\
\hline 20 & $M$ & 38 & Petroclival meningioma & 13.10.2017 & $\mathrm{D}$ & Y & $\mathrm{N}$ & $N$ \\
\hline 21 & $\mathrm{~F}$ & 26 & Rathke cleft cyst & 10.11.2017 & $\mathrm{D}$ & Y & $\mathrm{N}$ & $\mathrm{N}$ \\
\hline 22 & M & 21 & Cranipharyngioma & 29.12.2017 & D & Y & $\mathrm{N}$ & $\mathrm{N}$ \\
\hline 23 & M & 55 & Cranipharyngioma & 12.01.2018 & $D$ & Y & $\mathrm{N}$ & $\mathrm{N}$ \\
\hline
\end{tabular}

$F$-female, $M$ - male, $N C$ - cranial nerve, $D$-definite, I-indefinite, $Y$-yes, $N-$ no.

calization and character of a pathology determine the place and size of a dural defect, partially the intensity of CSF leakage as well as the level of difficulty of the skull base reconstruction. The literature review has shown that pituitary adenomas are the most common lesions, due to which EEA operations are performed. Kassam et al. reported a rate of $39.1 \%$ for pituitary adenomas in a group of $800 \mathrm{pa}-$ tients [6] and Hara et al. reported a rate of $63.4 \%$ for these tumors among 194 operations [8]. Thorp et al. found pituitary adenomas among $46 \%$ of 152 cases [9]. As far as the chordomas are concerned, their incidence rate in the above publications varied from $2.5 \%$ [6] to $6.7 \%$ [8]. The analysis of our own data revealed a much lower percentage of pituitary adenomas ( $28 \%$ of macroadenomas) and the prev- 


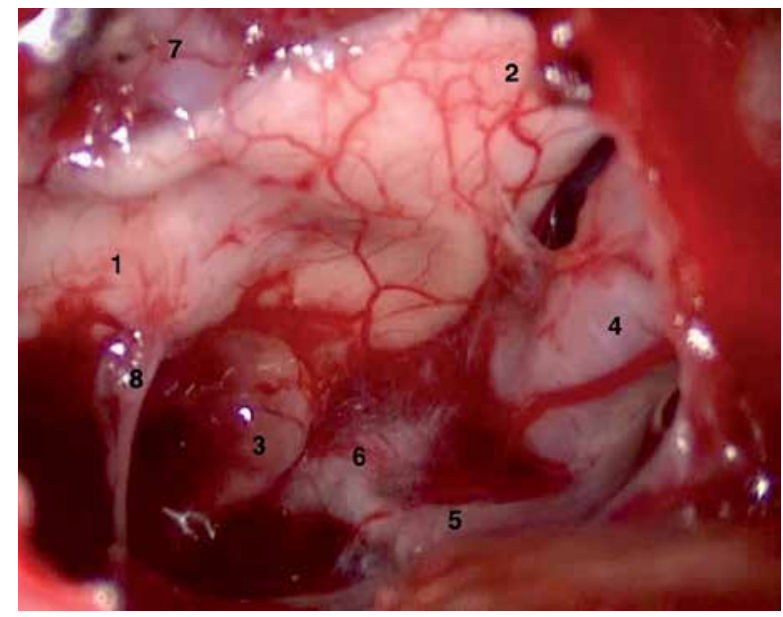

Photo 1. Tumor bed after resection of craniopharyngioma - intraoperative image (1 - optic chiasm, 2 - distal part of the left optic nerve, 3 - optic tract on the left-hand side, 4 - left internal carotid artery, 5 - left posterior communicating artery, 6 - left posterior cerebral artery, 7 - left anterior communicating artery, 8 -infundibulum)

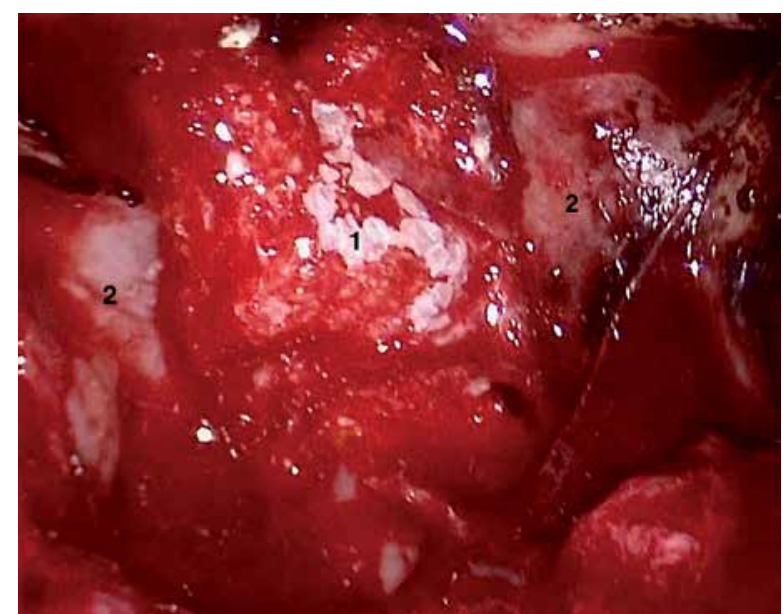

Photo 3. The next layer of the reconstruction (1 - fibrin sealant patch, 2 - the remaining posterior wall of the sphenoid sinus and Onodi cell on the left-hand side)

alence of other skull base tumors, with particular consideration of chordomas, which accounted for $28 \%$ of all lesions. Other pathologies (44\%) were: craniopharyngiomas, Rathke's cleft cyst, hemangiopericytoma and meningioma. It is our firm belief that due to the low number of presented cases, the results do not reflect the actual percentage of skull base tumors in the population. However, the authors emphasize that higher incidence of lesions

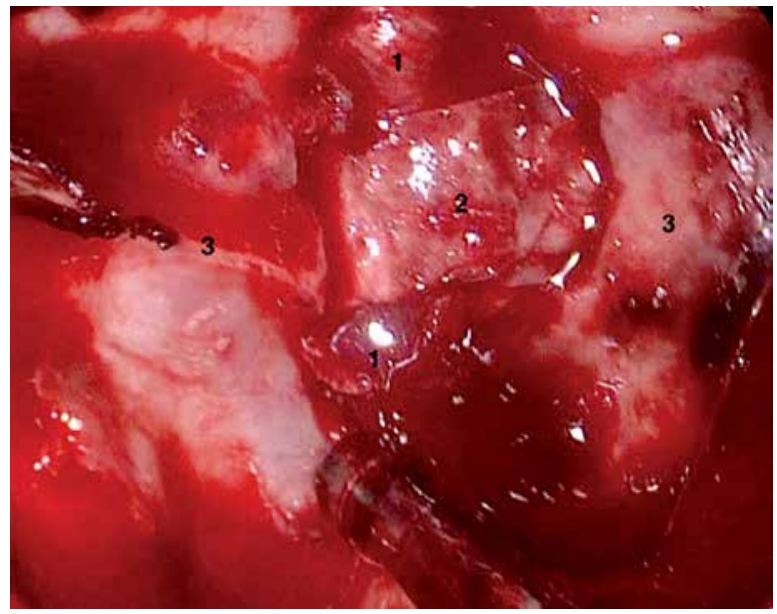

Photo 2. First layer of the reconstruction (1- rectus abdominis muscle fascia in an intradural localization, 2 - transverse bony trabecula (harvested at posterior septectomy) supporting the fascia, 3 - the remaining posterior wall of the sphenoid sinus and Onodi cell on the left-hand side)

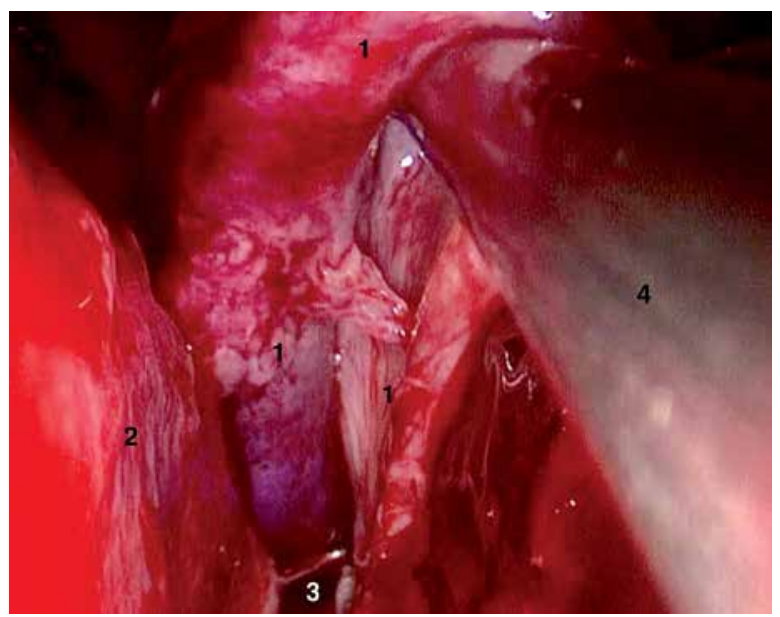

Photo 4. The final layer of the reconstruction (1 - nasoseptal flap (colored in violet) being repositioned from the nasopharynx, 2 - inferior nasal turbinate, 3 - choana, 4 - suction)

localized beyond the sella turcica could be a meaningful factor, which deteriorated the effectiveness of reconstruction.

The original method with the application of NSF has been widely modified. The modifications concerned various aspects of the flap harvesting, for instance, its donor size and side of its raising as well as planning the initial turbinectomy before NSF preparation. There are three main modifications 


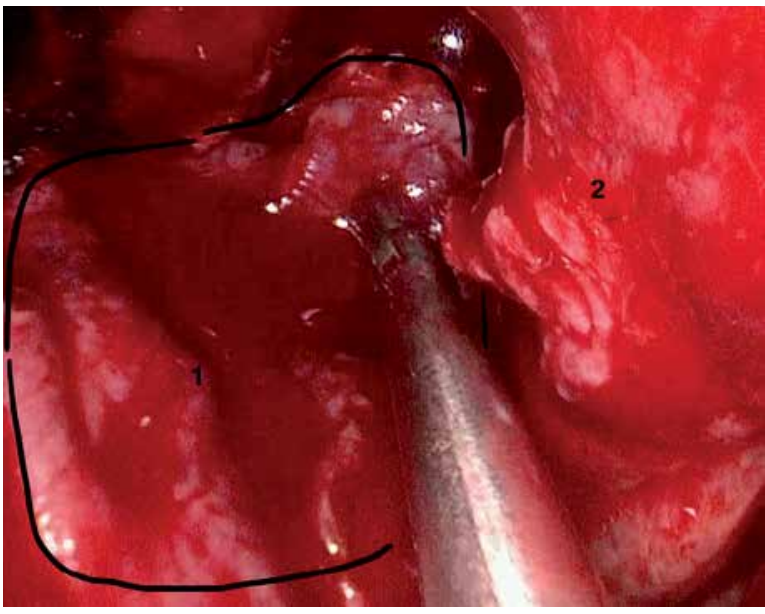

Photo 5. NSF positioned on the previous layers of the reconstruction ( 1 - the flap, 2 - nasal septum)

superior to the original technique which are worth mentioning.

The first one is applied in order to improve the remucosalization of the NSF donor site, i.e. the denuded nasal septum. It was introduced by CaicedoGranados et al. in 2010 [10]. It is formed of the nasal mucosa from the side opposite to the denuded septum. After posterior bony septectomy the flap is reversed $180^{\circ}$ and fixed to the donor site with a 4-0 absorbable stitch. The authors used the reversed rotation flap several times in cases of conducive anatomy, i.e. wide nostrils. When choosing the described technique it is worth considering that in spite of tight fixing of the reverse rotation flap to the donor site, the patency of the anterior part of the nostril might be significantly narrowed. Consequently, visualization of the more outlying lesions, for example of the clivus, may be deteriorated in the bimanual approach. Therefore, the authors, aware of their limited experience, used a reverse rotation flap only in cases of particularly conducive anatomy.

The second modification of the HBF is the 'rescue flap' method. It is intended for cases in which assessment of potential intraoperative CSF leakage is difficult to make. The removal of the anterior wall of the sphenoid sinus may be associated with damage of the posterior septal branches of the sphenopalatine artery (SPA), which might result in devascularization of the flap pedicle. Therefore, most frequently, if the lesion is located in the planum sphenoidale, sella turcica or clivus, the NSF is harvested at the onset of the operation and it is left in the nasopharynx for the

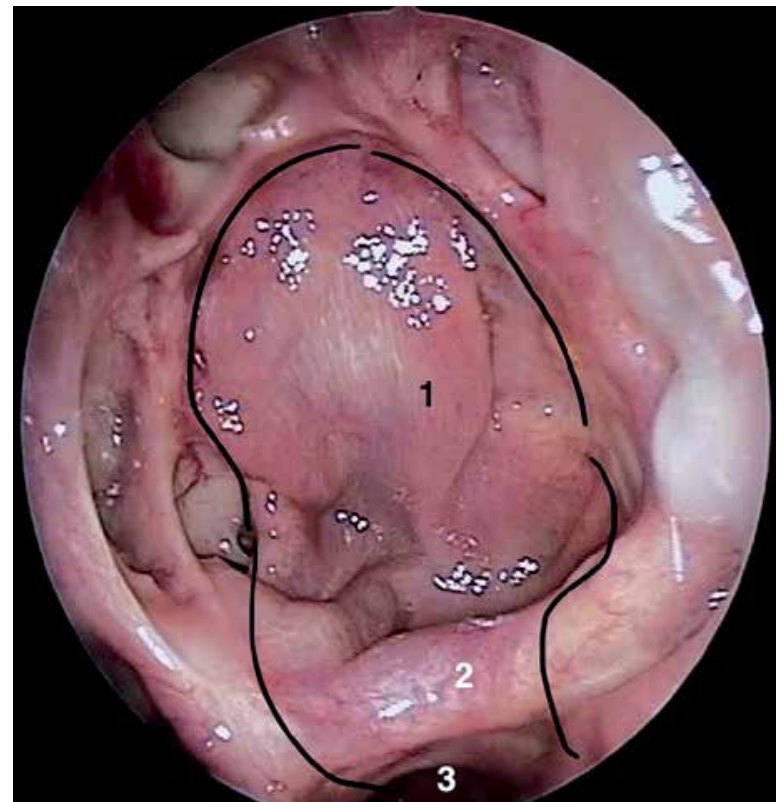

Photo 6. Healed NSF - postoperative image ( 1 - the main part of the flap covering the defect in the sella turcica and clivus, 2 - the flap pedicle, turning at the inferior wall of the sphenoid sinus, 3 - choana)

time of tumor resection. If the risk of CSF leakage is overestimated preoperatively, then an unnecessary procedure of harvesting the HBF is performed. The 'rescue flap' technique was introduced in order to avoid such situations [11]. In this approach the septal incisions are performed only in the most postero/superior part of the septum and sphenoid face until reaching the level of the floor (or below) of the sphenoid sinus. Then the mucosa below the sphenoid ostium, sphenoid rostrum, anterior wall of the sphenoid sinus and posterior part of the septum is elevated and displaced down. If no CSF leaks are encountered, the posterior mucosal flap is repositioned. If an intraoperative CSF leak is obtained, the rescue flap is then converted into a normal nasoseptal flap for skull base reconstruction by extending the incisions into standard NSF incisions [11]. The above rescue flap technique is particularly recommended for pituitary surgery, which in comparison to clival approaches does not require low opening of the sphenoid sinus. The authors of the present publication have successfully used the rescue flap technique in cases in which final NSF harvesting was unnecessary. At the same time, these cases were not included in the material of the described study. 
Another modification is a reverse harvesting sequence of the nasoseptal flap, which is recommended in cases with a severe septal spur [12]. The technique, reported by Corsten et al., allows elevation of the flap without mucosal tearing. Unlike in the standard NSF harvesting, the incisions are first performed on the contralateral to the donor site side of the septum. The reverse rotation flap is elevated anteriorly and, after cutting it off in the region of the sphenoid sinus, the septal spur is removed in retrograde fashion, preserving the mucosa of the NSF. Finally, the nasoseptal flap is raised in a conventional way [12]. According to the observations of other skull base surgeons, when choosing the side of NSF harvesting, it is recommended to take into consideration the lateralization of pathologies. In lateralized pathologies it is necessary to utilize the contralateral nasoseptal flap to reconstruct the skull base, with the ipsilateral reverse rotation flap used to reconstruct the septum. These spatial configurations provide safe reposition of the flap to the target place without extensive angulation of its pedicle or deterioration of the blood supply. The authors have not used the above technique, as there were no cases of lateralized tumor with a contralateral septal spur that could result in perforation of the mucosa during elevation of the mucoperichondrial flap.

The standard protocol of NSF harvesting requires its preparation prior to tumor dissection in order to avoid any damage of its vascularized pedicle when performing a transsphenoidal approach. For the time of tumor resection, the flap is left in the nasopharynx or maxillary sinus. A risk of unnecessary flap harvesting exists. In case of lack of anticipated intraoperative CSF leakage, HBF harvesting remains redundant and it leads to prolonged healing and higher morbidity of the procedure. Therefore, the authors claim that meticulous guidelines for NSF harvesting would minimize the risk of unnecessary flap preparation. Absolute and relative indications for HBF harvesting, mentioned above, have been defined. The indications for dissection of pituitary macroadenoma of more than $2.5 \mathrm{~cm}$ in diameter were based on observations made by Jakimovski et al. [7] and Schwartz et al. Considering the described criteria, among 25 EEA operations with HBF application, 13 absolute and 12 relative indications were assessed preoperatively. The evaluation appeared to be correct in 23 cases. In 2 cases, in both of which the indications were relative, there was no
CSF leak. Therefore, NSF harvesting was redundant and it was replaced to the donor site on the septum. Early harvesting of the vascularized nasoseptal flap was also reported by Eloy et al. [13]. They claim that correct evaluation of anticipated CSF leak and appropriate qualification for HBF harvesting is possible if meticulous verification of diagnostic imaging is performed. They recommend NSF preparation in the following cases: intradural pathology, extradural pathology with dural and intradural involvement, and pituitary adenoma with anticipated large skull base and dural defects. They report only one case (out of 87 patients) in which the skull base defect was not repaired with the harvested NSF, because a highflow intraoperative CSF leak was not encountered. Inclusion criteria presented in the study of Eloy et al. are similar to our own. However, we believe that our relative criteria are of higher accuracy and that they are established with particular consideration of other clinical factors, such as reoperations. The worse correctness of the qualification in our study is a result of poorer experience despite very strict planning of the surgery.

As far as the lumbar drainage is concerned, there are no unequivocal guidelines regulating its application in skull base reconstructive surgery. Several surgeons supported most of their patients with a lumbar drain preoperatively, for instance McCoul et al. in $82.4 \%$ of patients [14]. According to the cited authors any time an indication for NSF application in the resection of macroadenomas (described above) is found, lumbar drainage should be performed. Others place a lumbar drain only if a high-volume CSF leak is encountered intraoperatively during the primary operation [6] or postoperatively [15]. In the case of a postoperative CSF leak Zanation et al. recommend prompt reoperation and assessment of the CSF volume. If at that time high-flow leakage is noted, spinal lumbar drainage is considered [16]. The authors of the present study used lumbar drainage only in one case of postoperative CSF leakage, which was performed together with reoperation.

Complications in EEA operations with HBF occur in several percentage of cases. The most common failure is CSF leakage. Thorp et al. report CSF leakage in $3.3 \%$ of cases among 144 operations with NSF [9], Liu et al. reported 3.2\% in a group of 93 patients [17] and Kassam et al. reported 6\% [6]. In the present study there was only one case of postoperative CSF leakage, which accounts for $4 \%$ of all cases. Anoth- 
er complication that might be encountered is flap necrosis, which sometimes is a nidus for infection [18]. According to Chabot et al. necrotic NSF was found in 8 out of 601 patients. The authors mention that signs of meningitis after EEA in the absence of a clear CSF leak with lack of NSF enhancement on MRI should raise the suspicion of necrotic NSF. These patients require prompt exploration and debridement of nonviable tissue with revision of skull base reconstruction [18]. In our study, similarly to the data reported by Thorp et al., no nasoseptal flap necrosis was observed [9]. Harvey et al., who made a systemic review of publications about endoscopic skull base reconstructions of large dural defects, revealed that apart from CSF leaks and flap necrosis, pneumocephalus, epistaxis, intracranial bleeding, meningitis, sinusitis and pulmonary embolus/deep vein thrombus were also reported [19].

The main reasons for complications within the flap itself identified by different authors most frequently concern:

- Too little flap or too narrow margin of the flap placed on the recipient site,

- Tucking of flap margins,

- Reverse placement of the flap,

- Pedicle constriction, ischemia of the flap,

- Incomplete denudation of the bony margin of the reconstructed defect,

- No adhesion of the flap pedicle or its retraction,

- Ineffective arrangement of the superior layers of the multilayer reconstruction.

Our complication was associated with the latter reason. The imperfect algorithm of the initial layers of the skull base reconstruction after resection of the clival chordoma precluded sealing of the CSF leak. Consequently, it prevented early adhesion of the flap and led to preservation of the fistula. The patient was reoperated on and the primary flap was supported by abdominal fat and fascia of the rectus abdominis muscle. Lumbar drainage was also performed in this case. However, it is not routinely used by the authors. Complete healing was observed.

\section{Conclusions}

Skull base reconstruction with HBF is a highly effective technique. It gives a relatively low risk of complications despite limited experience of the operating team. Due to several nuances of the procedure, it is crucial to define appropriate criteria of qualification for NSF harvesting prior to resection in order to avoid redundant flap raising, which increases the morbidity of the operation. Various modifications of the standard technique partially solve its basic drawbacks. However, they require high experience and good surgical skills for its successful application. Absolute indications for NSF harvesting prior to resection are: reoperations in the case of a previous open approach, preoperative CSF leakage, intradural localization of a tumor related to its etiopathogenesis, and suspicion of intradural diffusion of a neoplasm in MRI if the etiopathogenesis cannot clarify the tumor's relation to the meninges (mainly chordoma). Relative indications concern mostly pituitary macroadenomas of at least $2.5 \mathrm{~cm}$ in diameter assessed according to the Schwartz classification and localization. Further studies on a larger number of patients should be performed.

\section{Conflict of interest}

The authors declare no conflict of interest.

\section{References}

1. Hirsch O. Successful closure of cerebrospinal fluid rhinorrhea by endonasal surgery. AMA Arch Otolaryngol 1952; 56: 1-13.

2. Wigand M. Transnasal ethmoidectomy under endoscopical control. Rhinology 1981; 19: 7-15.

3. Patel MR, Taylor RJ, Hackman TG, et al. Beyond the nasoseptal flap: outcomes and pearls with secondary flaps in endoscopic endonasal skull base reconstruction. Laryngoscope 2014; 124: 846-52.

4. Hadad G, Bassagasteguy L, Carrau RL, et al. A novel reconstructive technique after endoscopic expanded endonasal approaches: vascular pedicle nasoseptal flap. Laryngoscope 2006; 116: 1882-6.

5. Bhatki AM, Carrau RL, Snyderman CH, et al. Endonasal surgery of the ventral skull base: endoscopic transcranial surgery. Oral Maxillofac Surg Clin North Am 2010; 22: 157-68.

6. Kassam AB, Prevedello DM, Carrau RL et al. Endoscopic endonasal skull base surgery: analysis of complications in the authors' initial 800 patients. J Neurosurg 2011; 114: 1544-68.

7. Jakimovski D, Bonci G, Attia M, et al. Incidence and significance of intraoperative cerebrospinal fluid leak in endoscopic pituitary surgery using intrathecal fluorescein. World Neurosurg 2014; 82: 513-23.

8. Hara T, Akutsu H, Yamamoto T, et al. Cranial base repair using suturing technique combined with a mucosal flap for cerebrospinal fluid leakage during endoscopic endonasal surgery. World Neurosurg 2015; 84: 1887-93.

9. Thorp BD, Sreenath SB, Ebert CS, et al. Endoscopic skull base reconstruction: a review and clinical case series of 152 vascularized flaps used for surgical skull base defects in the setting of intraoperative cerebrospinal fluid leak. Neurosurg Focus 2014; 37: E4. 
10. Caicedo-Granados E, Carrau R, Snyderman CH, et al. Reverse rotation flap for reconstruction of donor site after vascular pedicled nasoseptal flap in skull base surgery. Laryngoscope 2010; 120: 1550-2.

11. Rivera-Serrano CM, Snyderman CH, Gardner P, et al. Nasoseptal "rescue" flap: a novel modification of the nasoseptal flap technique for pituitary surgery. Laryngoscope 2011; 121: 990-3.

12. Corsten M, Kassam A, Al-Mutairi D, et al. Reverse harvesting sequence of nasoseptal flaps during endoscopic skull base surgery: technical modification to deal with the severe septal spur. Laryngoscope 2013; 123: 73-5.

13. Eloy JA, Patel AA, Shukla PA, et al. Early harvesting of the vascularized pedicled nasoseptal flap during endoscopic skull base surgery. Am J Otolaryngol 2013; 34: 188-94.

14. McCoul ED, Anand VK, Singh A, et al. Long-term effectiveness of a reconstructive protocol using the nasoseptal flap after endoscopic skull base surgery. World Neurosurg 2014; 81: 136-43.

15. Gardner PA, Kassam AB, Snyderman CH, et al. Outcomes following endoscopic, expanded endonasal resection of suprasellar craniopharyngiomas: a case series. J Neurosurg 2008; 109: 6-16.

16. Zanation AM, Thorp BD, Parmar P. Reconstructive options for endoscopic skull base surgery. Otolaryngol Clin North Am 2011; 44: 1201-22.

17. Liu JK, Schmidt RF, Choudhry OJ, et al. Surgical nuances for nasoseptal flap reconstruction of cranial base defects with highflow cerebrospinal fluid leaks after endoscopic skull base surgery. Neurosurg Focus 2012; 32: E7.

18. Chabot JD, Patel CR, Hughes MA, et al. Nasoseptal flap necrosis: a rare complication of endoscopic endonasal surgery. J Neurosurg 2017; 21: 1-10.

19. Harvey RJ, Parmar P, Sacks R, et al. Endoscopic skull base reconstruction of large dural defects: a systematic review of published evidence. Laryngoscope 2012; 122: 452-9.

Received: 12.10 .2018 , accepted: 6.05.2019. 